\title{
MEASURES WITH UNIQUE TANGENT MEASURES IN METRIC GROUPS
}

\author{
PERTTI MATTILA
}

\begin{abstract}
We show that a Radon measure on a locally compact metric group with natural dilations has almost everywhere a unique tangent measure if and only if it has almost everywhere a Haar measure of a closed dilation invariant subgroup as its unique tangent measure.
\end{abstract}

\section{Introduction}

If a measure in $\mathrm{R}^{n}$ around almost every point looks essentially the same at all small scales, how does it look like? The answer, which is not so suprising, is that it looks like the $m$-dimensional Lebesgue measure on an $m$-plane, where $m$ can vary from point to point. We observed with David Preiss about ten years ago that this can be deduced from some results in [7]. Here I address the same question in a general setting. A particular motivation comes from a mysterious question: what should rectifiability mean in Heisenberg groups and in more general Carnot-Carathéodory groups? We shall comment on this later a little more but here let us just look at it from the point of view of rectifiability in $\mathbf{R}^{n}$.

For subsets of $\mathbf{R}^{n}$ with finite $m$-dimensional Hausdorff measure there are many different characterizations of rectifiability, see [4] and [6], but one way of thinking about it is that rectifiability should be opposite to any kind of fractality. Fractality could mean that landscapes keep changing when you go deeper and deeper into the set (or measure) while for rectifiable objects they approach a single view.

Taking the last point of view as a starting point we shall study rectifiability in a general setting of a locally compact group with natural dilations and we use the notion of tangent measure in the sense of Preiss [7] to define the limiting views. The main result roughly says that in such a setting if a Radon measure has at almost every point a unique tangent measure, then at almost every point it has a Haar measure of a closed dilation invariant subgroup as its unique tangent measure.

Received December 8, 2004. 


\section{Groups, dilations, and tangent measures}

Throughout the rest of this paper we assume that $G$ is a locally compact metric group with the following properties:

(2.1) The left translations

$$
\tau_{a}: x \rightarrow a^{-1} x, x \in G,
$$

are isometries for all $a \in G$.

(2.2) There are dilations

$$
\delta_{r}: G \rightarrow G, r>0,
$$

which are continuous group homomorphisms and which satisfy

(i) $\delta_{1}=$ identity,

(ii) $d\left(\delta_{r}(x), \delta_{r}(y)\right)=r d(x, y)$ for $x, y \in G, r>0$,

(iii) $\delta_{r s}=\delta_{r} \circ \delta_{s}$ for $r, s>0$.

Then $\delta_{r}$ is an isomorphism with

$$
\delta_{r}^{-1}=\delta_{1 / r} .
$$

The map

$$
T_{a, r}=\delta_{1 / r} \circ \tau_{a}, \quad a \in G, r>0,
$$

maps the closed ball $B(a, r)$ with centre a and radius $r$ onto the closed unit ball $B(0,1)$. (0 is the identity element of $G$.)

The image $f_{\#} \mu$ of a measure $\mu$ on $G$ under a map $f: G \rightarrow G$ is defined by

$$
f_{\#} \mu(A)=\mu\left(f^{-1}(A)\right) \quad \text { for } \quad A \subset G .
$$

A measure always means an outer measure. A Radon measure on $G$ means a locally finite Borel regular outer measure on $G$. This is in accordance with other usual definitions.

Definition 2.3. Let $\mu$ be a Radon measure on $G$. We say that $v$ is a tangent measure of $\mu$ at $a \in G$ if $\nu$ is a Radon measure on $G$ with $v(G)>0$ and there are positive numbers $c_{i}$ and $r_{i}, i=1,2, \ldots$, such that $r_{i} \rightarrow 0$ and

$$
c_{i} T_{a, r_{i} \#} \mu \rightarrow v \quad \text { weakly as } i \rightarrow \infty .
$$

We denote by $\operatorname{Tan}(\mu, a)$ the set of all tangent measures of $\mu$ at $a$.

The numbers $c_{i}$ are normalization constants which are needed to keep $v$ non-trivial and locally finite. Often, as below, one can use $c_{i}=\mu\left(B\left(a, r_{i}\right)\right)^{-1}$. 
Definition 2.4. Let $\mu$ be a Radon measure on $G$. We say that $\mu$ has a unique tangent measure $v$ at $a$ if $v$ is a Radon measure on $G$ such that

$$
\operatorname{Tan}(\mu, a)=\{c v: 0<c<\infty\} .
$$

Of course, such a $v$ is unique only up to multiplication by positive constants.

Lemma 2.5. Let $\mu$ be a Radon measure on $G$. If $\mu$ has a unique tangent measure $v$ at $a$, then there are $\alpha \geq 0$ and $c>0$ such that

(1) $v(B(0, \varrho))=v(B(0,1)) \varrho^{\alpha}$ for $\varrho>0$,

(2) $\mu(B(a, r))^{-1} T_{a, r \#} \mu \rightarrow c v$ weakly as $r \rightarrow 0$, and

(3) $\lim _{r \rightarrow 0} \frac{\mu(B(a, \varrho r))}{\mu(B(a, r))}=\varrho^{\alpha}$ for $\varrho>0$.

Proof. Obviously $\delta_{\varrho \#} v \in \operatorname{Tan}(\mu, a)$ for all $\varrho>0$, whence, by the uniqueness of $\nu$, there is $c(\varrho)>0$ such that

$$
\delta_{\varrho \#} v=c(\varrho) v .
$$

Then we also have for $\varrho, \sigma>0$,

$$
c(\varrho \sigma) v=\delta_{\varrho \sigma \#} \nu=\delta_{\varrho \#} \delta_{\sigma \#} \nu=c(\varrho) c(\sigma) v,
$$

consequently,

$$
c(\varrho \sigma)=c(\varrho) c(\sigma) .
$$

From (2.6) and (2.2) we see that

$$
c(\varrho)=\frac{v(B(0,1))}{v(B(0, \varrho))}
$$

for all $\varrho>0$. Clearly, $c$ is a decreasing function which together with (2.7) implies that there is $\alpha \geq 0$ such that

$$
c(\varrho)=\varrho^{-\alpha} \quad \text { for } \quad \varrho>0 .
$$

This gives (1) by (2.8).

From (1) we conclude that $v(\partial B(0, \varrho))=0$ for all $\varrho>0$ and therefore, when $v=\lim _{i \rightarrow \infty} c_{i} T_{a, r_{i} \#} \mu$,

$$
\varrho^{\alpha}=\frac{v(B(0, \varrho))}{v(B(0,1))}=\frac{\lim _{i \rightarrow \infty} c_{i} \delta_{1 / r_{i} \#} \tau_{a \#} \mu(B(0, \varrho))}{\lim _{i \rightarrow \infty} c_{i} \delta_{1 / r_{i} \#} \tau_{a \#} \mu(B(0,1))}=\lim _{i \rightarrow \infty} \frac{\mu\left(B\left(a, \varrho r_{i}\right)\right)}{\mu\left(B\left(a, r_{i}\right)\right)} .
$$


Since this holds for all $\varrho>0$, we get for all $p \in \mathbf{Z}$ that

$$
\lim _{i \rightarrow \infty} \frac{\mu\left(B\left(a, 2^{p+1} r_{i}\right)\right)}{\mu\left(B\left(a, 2^{p-1} r_{i}\right)\right)}=4^{\alpha} .
$$

Next we check that

$$
\limsup _{r \rightarrow 0} \frac{\mu(B(a, 2 r))}{\mu(B(a, r))}<\infty .
$$

From (2.9) we see that for all $j=1, \ldots$ there is $i_{j}$ such that

$$
\mu\left(B\left(a, 2^{1-p} r_{i_{j}}\right)\right)<4^{\alpha+1} \mu\left(B\left(a, 2^{-1-p} r_{i_{j}}\right)\right) \quad \text { for } \quad p=0,1, \ldots, j,
$$

whence

$$
\mu(B(a, 2 r))<4^{\alpha+1} \mu(B(a, r)) \quad \text { for } \quad 2^{-j} r_{i_{j}}<r<r_{i_{j}} .
$$

By approximation this holds also for open balls $U(a, r)$ :

$$
\mu(U(a, 2 r))<4^{\alpha+1} \mu(U(a, r)) \quad \text { for } \quad 2^{-j} r_{i_{j}}<r<r_{i_{j}} .
$$

Let

$$
s_{j}=\inf \left\{s: 0<s<r_{i_{j}}, \mu(U(a, 2 r))<4^{\alpha+1} \mu(U(a, r)) \text { for } s<r<r_{i_{j}}\right\} .
$$

If $s_{j}=0$ for some $j$ we get (2.10). Otherwise $s_{j}>0$ for all $j$,

$$
\mu(U(a, 2 r))<4^{\alpha+1} \mu(U(a, r)) \quad \text { for } \quad s_{j}<r<r_{i_{j}},
$$

and

$$
\mu\left(a, U\left(2 s_{j}\right)\right) \geqslant 4^{\alpha+1} \mu\left(U\left(a, s_{j}\right)\right) .
$$

By (2.11) and (2.13), $s_{j} / r_{i_{j}} \rightarrow 0$ as $j \rightarrow \infty$. Thus by (2.12) and the weak compactness of measures the sequence $\left(s_{j}\right)$ has a subsequence $\left(s_{j_{k}}\right)$ such that

$$
\frac{1}{\mu\left(U\left(a, 2 s_{j_{k}}\right)\right)} T_{a, s_{j_{k}} \#} \mu \rightarrow \lambda
$$

where $\lambda$ is a tangent measure of $\mu$ at $a$. Then

$$
\lambda(B(0,2)) \geqslant \limsup _{k \rightarrow \infty} \frac{\mu\left(B\left(a, 2 s_{j_{k}}\right)\right)}{\mu\left(U\left(a, 2 s_{j_{k}}\right)\right)} \geqslant 1,
$$


and by (2.13)

$$
\lambda(U(0,1)) \leqslant \liminf _{k \rightarrow \infty} \frac{\mu\left(U\left(a, s_{j_{k}}\right)\right)}{\mu\left(U\left(a, 2 s_{j_{k}}\right)\right)} \leqslant 4^{-\alpha-1} .
$$

This shows by (1) that $\lambda$ cannot be a constant multiple of $v$, gives a contradiction and proves (2.10).

From (2.10) we get by the weak compactness of measures that every sequence $\left(\mu\left(B\left(a, r_{i}\right)\right)^{-1} T_{a, r_{i} \#} \mu\right)$ with $r_{i} \rightarrow 0$ has a converging subsequence. By the uniqueness of the tangent measure at $a$, this gives (2), and consequently also (3) by the above argument.

Let $\lambda$ be a left Haar measure of $G$. Then $\lambda$ is uniformly distributed in the sense that the measures of the balls only depend on the radius:

$$
\lambda(B(x, r))=\lambda(B(0, r)) \quad \text { for } \quad x \in G, r>0 .
$$

This follows immediately from (2.1) since $B(a, r)=\tau_{a^{-1}}(B(0, r))$.

Proposition 2.14. Let $\lambda$ be a left Haar measure of G. Then there is $\alpha \geqslant 0$ such that for all $a \in G$ and $r>0$,

(1) $\lambda(B(a, r))=\lambda(B(0,1)) r^{\alpha}$.

Proof. Since the dilations $\delta_{r}$ and translations $\tau_{a}$ are homeomorphisms we get from the local compactness that all closed balls of $G$ are compact. Hence there is an integer $N$ such that $B(0,2)$ can be covered with $N$ balls of radius 1. Using the dilations and translations we obtain that any ball $B(a, 2 r)$ can be covered with $N$ balls $B\left(a_{1}, r\right), \ldots, B\left(a_{N}, r\right)$. Since $\lambda\left(B\left(a_{i}, r\right)\right)=\lambda(B(a, r))$, we get

$$
\lambda(B(a, 2 r)) \leqslant \sum_{i=1}^{N} \lambda\left(B\left(a_{i}, r\right)\right)=N \lambda(B(a, r)) .
$$

This implies that $\operatorname{Tan}(\lambda, a) \neq \emptyset$ for all $a \in G$. Since $\tau_{b \#} \lambda=\lambda$ for all $b \in G$, we see easily that all tangent measures of $\lambda$ are left invariant, whence constant multiples of $\lambda$. Then (1) follows from Lemma 2.5(1).

Next we present Preiss's principle "tangent measures to tangent measures are tangent measures" in our setting. We denote by spt $v$ the support of a measure $v$.

Proposition 2.15. Let $\mu$ be a Radon measure on $G$ such that

(1) $\limsup _{r \rightarrow 0} \frac{\mu(B(a, 2 r))}{\mu(B(a, r))}<\infty$ for $\mu$ almost all $a \in G$.

Then for $\mu$ almost all $a \in G$, for all $v \in \operatorname{Tan}(\mu, a)$, 
(2) $T_{x, \varrho \# ~} v \in \operatorname{Tan}(\mu, a)$ for $x \in \operatorname{spt} v, \varrho>0$,

(3) $\operatorname{Tan}(v, x) \subset \operatorname{Tan}(\mu, a)$ for $x \in \operatorname{spt} v$.

The proof in $\mathrm{R}^{n}$ is given in [7] and also (essentially the same) in [6]. Since only small changes are required in the present setting, we explain them following the presentation of [6] without giving all the details.

We need two things about weak convergence. The first is that it is given by the metrics $F_{r}, r>0$, as in [6, Lemma 14.13]. The proof is the same. It uses that continuous functions can be uniformly approximated by Lipschitz functions on compact sets which is, for example, a consequence of the StoneWeierstrass approximation theorem. Recall that all closed balls are compact. Secondly, we need that the space of Radon measures on $G$ is weakly separable, which is an easy exercise.

Then we check the proof of [6, Theorem 14.16]. We need to check more carefully some parts due to the fact that $\tau_{a}$ and $\delta_{r}$ need not commute in the same way as in $\mathrm{R}^{n}$. So as in [6] we have $A \subset G$ with $\mu(A)>0$ and for $a \in A$ we have

$$
v_{a}=\lim _{i \rightarrow \infty} c_{i} T_{a, r_{i} \#} \mu \in \operatorname{Tan}(\mu, a)
$$

and

$$
x_{a} \in \operatorname{spt} v_{a}
$$

such that (3), (4) and (5) on page 197 of [6] hold. (5) follows from the density theorem for $\mu$ which is a consequence of our assumption (1). Hence, as in [6], we find $a_{i} \in A$ such that, using also (2.1) and (2.2),

$$
d\left(x_{a}, \delta_{1 / r_{i}}\left(a^{-1} a_{i}\right)\right)=d\left(\delta_{r_{i}}\left(x_{a}\right), a^{-1} a_{i}\right) / r_{i}=d\left(a \delta_{r_{i}}\left(x_{a}\right), a_{i}\right) / r_{i} \rightarrow 0 .
$$

Using (2.16) we compute (the change from a double limit to a single limit is an easy exercise)

$$
\begin{aligned}
\tau_{x_{a} \#} v_{a}=\lim _{i \rightarrow \infty} \tau_{\delta_{1 / r_{i}}\left(a^{-1} a_{i}\right) \#}\left(c_{i} T_{a, r_{i} \# \mu}\right) & =\lim _{i \rightarrow \infty} c_{i}\left(\tau_{\delta_{1 / r_{i}}\left(a^{-1} a_{i}\right)} \circ \delta_{1 / r_{i}} \circ \tau_{a}\right)_{\#} \mu \\
& =\lim _{i \rightarrow \infty} c_{i} T_{a_{i}, r_{i} \#} \mu,
\end{aligned}
$$

because

$$
\begin{aligned}
\tau_{\delta_{1 / r_{i}}\left(a^{-1} a_{i}\right)} \circ \delta_{1 / r_{i}} \circ \tau_{a}(x) & =\left(\delta_{1 / r_{i}}\left(a^{-1} a_{i}\right)\right)^{-1} \delta_{1 / r_{i}}\left(a^{-1} x\right) \\
& =\delta_{1 / r_{i}}\left(a_{i}^{-1} a\right) \delta_{1 / r_{i}}\left(a^{-1} x\right) \\
& =\delta_{1 / r_{i}}\left(a_{i}^{-1} x\right)=T_{a_{i}, r_{i}}(x),
\end{aligned}
$$

since $\delta_{1 / r_{i}}$ is a homomorphism. After this the proof goes exactly as in [6]. 


\section{Measures with unique tangent measures}

First we give a characterization of left Haar measures on $G$.

Proposition 3.1. Let $\lambda$ be a Radon measure on $G$. Then $\lambda$ is a left Haar measure on $G$ if and only if for all $a \in G$ and all $r>0$ there are positive numbers $c(a)$ and $d(r)$ such that

(1) $\tau_{a \#} \lambda=c(a) \lambda$ and

(2) $\delta_{r \# \lambda}=d(r) \lambda$.

REMARK. Measures satisfying (1) have been studied in [4] in a more general setting. Federer called them covariant and gave a characterization which we could also use. But since this would save only a few lines, we give the full proof below in our metric setting.

Proof OF Proposition 3.1. First, if $\lambda$ is a left Haar measure it satisfies (1) with $c(a)=1$. Since $\delta_{1 / r}=\delta_{r}^{-1}$ we have for all $a \in G, r>0$ and $\sigma>0$,

$$
\delta_{r \#} \lambda(B(a, \sigma))=\lambda\left(B\left(\delta_{1 / r}(a), \sigma / r\right)\right)=\lambda(B(0, \sigma / r))=\delta_{r \#} \lambda(B(0, \sigma)) .
$$

Hence $\delta_{r \#} \lambda$ is uniformly distributed. Since also $\lambda$ is uniformly distributed and such measures are uniquely determined up to multiplication by constants, see [6, Theorem 3.4], we get (2).

Next we prove the converse. Set

$$
\chi(a)=c\left(a^{-1}\right) \quad \text { for } \quad a \in G .
$$

As in the proof of Lemma 2.5, $\chi$ is a homomorphism. It is also easy to check that it is continuous. Define a Radon measure $v$ on $G$ by

$$
v(A)=\int_{A} \chi d \lambda
$$

for Borel sets $A \subset G$. Hence

$$
\lambda(A)=\int_{A} \chi^{-1} d \nu
$$

Then for any continuous function $\varphi$ on $G$ with compact support and for any $a \in G$,

$$
\begin{aligned}
\int \varphi \circ \tau_{a} d \nu & =\int \chi\left(\varphi \circ \tau_{a}\right) d \lambda=\frac{1}{\chi\left(a^{-1}\right)} \int \chi\left(a^{-1} x\right) \varphi\left(a^{-1} x\right) d \lambda x \\
& =\frac{1}{c(a)} \int(\chi \varphi) \circ \tau_{a} d \lambda=\frac{1}{c(a)} \int \chi \varphi d \tau_{a \#} \lambda \\
& =\int \chi \varphi d \lambda=\int \varphi d \nu .
\end{aligned}
$$


So $v$ is a left Haar measure on $G$. By Proposition 2.14 we know that there is $\alpha \geq 0$ such that, with $c=\lambda(B(0,1))$,

$$
v(B(x, r))=c r^{\alpha} \quad \text { for } \quad x \in G, r>0 .
$$

By (2) for $\varrho>0, r>0, y \in G$,

$$
\begin{aligned}
\frac{d(1 / \varrho)}{v(B(0, r))} \int_{B(y, r)} \chi^{-1} d v & =\frac{d(1 / \varrho) \lambda(B(y, r))}{v(B(0, r))} \\
& =\frac{\delta_{1 / \varrho \# \lambda(B(y, r))}^{v(B(0, r))}}{} \\
& =\frac{\lambda\left(B\left(\delta_{\varrho}(y), \varrho r\right)\right)}{v(B(0, r))} \\
& =\varrho^{\alpha} \frac{1}{v(B(0, \varrho r))} \int_{B\left(\delta_{\varrho}(y), \varrho r\right)} \chi^{-1} d \nu .
\end{aligned}
$$

Letting $r \rightarrow 0$ we get

$$
d(1 / \varrho) \chi(y)^{-1}=\varrho^{\alpha} \chi\left(\delta_{\varrho}(y)\right)^{-1},
$$

Taking $y=0$ we have $d(1 / \varrho)=\varrho^{\alpha}$ so that

$$
\chi(y)=\chi\left(\delta_{\varrho}(y)\right) \quad \text { for } \quad y \in G, \varrho>0 .
$$

Letting $\varrho \rightarrow 0$ we obtain

$$
\chi(y)=\chi(0) \quad \text { for } \quad y \in G,
$$

from which the proposition follows.

Now we can prove the main result of the paper.

Theorem 3.2. Let $\mu$ be a Radon measure on $G$. Then the following two conditions are equivalent:

(1) $\mu$ has a unique tangent measure $v_{a}$ at $\mu$ almost all points $a \in G$.

(2) For $\mu$ almost all $a \in G$ there exists a closed subgroup $H_{a}$ of $G$ which is invariant under the dilations $\delta_{r}, r>0$, and for which

$$
\operatorname{Tan}(\mu, a)=\left\{c \lambda_{a}: 0<c<\infty\right\}
$$

where $\lambda_{a}$ is a left Haar measure of $H_{a}$. 
Proof. Of course, (2) implies (1). Suppose (1) holds. By Lemma 2.5(3) and Proposition 2.15 for $\mu$ almost all $a \in G$ there are for all $x \in \operatorname{spt} v_{a}$ and for all $r>0$ positive numbers $c(x)$ and $d(r)$ such that

$$
\tau_{x \# v_{a}}=c(x) v_{a}
$$

and

$$
\delta_{r \#} v_{a}=d(r) v_{a} .
$$

This yields easily that $H_{a}:=\operatorname{spt} v_{a}$ is a closed subgroup of $G$ which is invariant under the dilations $\delta_{r}$. Hence we can apply Proposition 3.1 to conclude that $v_{a}$ is a left Haar measure on $H_{a}$. This proves the theorem.

\section{Heisenberg group}

As an example we first look at the lowest dimensional Heisenberg group

$$
\mathrm{H}^{1}=\mathrm{C} \times \mathrm{R}
$$

with the group law

$$
(w, s) \cdot(z, t)=(w+z, s+t+2 \operatorname{Im} w \bar{z}) .
$$

The dilations $\delta_{r}$ are given by

$$
\delta_{r}(z, t)=\left(r z, r^{2} t\right) .
$$

A natural metric $d$ on $\mathrm{H}^{1}$ is defined by

$$
d(P, Q)=\left\|P^{-1} \cdot Q\right\|,
$$

where

$$
\|P\|=\max \left\{|z|,|t|^{\frac{1}{2}}\right\} \quad \text { for } \quad P=(z, t) \in \mathrm{H}^{1} .
$$

Then (2.1) and (2.2) hold for $G=\mathrm{H}^{1}$.

The Hausdorff dimension of $\mathrm{H}^{1}$ is 4 . The lower dimensional (in the sense of Hausdorff dimension) closed subgroups, which are invariant under the dilations, are rather easily found. The 1-dimensional ones are the horizontal lines, that is, the lines in $\mathrm{C} \times\{0\}$ through the origin. There is only one 2-dimensional, the vertical line

$$
L_{V}=\{(0, t): t \in \mathrm{R}\} .
$$

The 3-dimensional ones are the vertical planes, that is, the cartesian products $L \times L_{V}$ where $L$ is a horizontal line.

So Theorem 3.2 tells us that if we have a Radon measure in $\mathrm{H}^{1}$ with the unique tangent measure property as in Theorem 3.2 it has to look locally around 
almost every point like one of the above alternatives, or like the Lesbegue measure of $\mathrm{R}^{3}$ (which is the same as the 4-dimensional Hausdorff measure and also a Haar measure of the group), or like the Dirac delta at 0. Of course, its appearance can change from point to point.

How is this related to rectifiability? Not much if we take the usual definition of rectifiability as in [4]: a set $E \subset \mathrm{H}^{1}$ is $k$-rectifiable if there are Lipschitz maps $f_{i}: \mathrm{R}^{k} \rightarrow \mathrm{H}^{1}$ such that

$$
\mathscr{H}^{k}\left(E \backslash \bigcup_{i=1}^{\infty} f_{i}\left(\mathrm{R}^{k}\right)\right)=0
$$

where $\mathscr{H}^{k}$ is the $k$-dimensional Hausdorff measure (of course, defined with the metric $d$ ). Ambrosio and Kirchheim showed in [1] that there are no $k$ rectifiable sets in $\mathrm{H}^{1}$ with positive $\mathscr{H}^{k}$ measure for $k=2$ and 3 . For more general results of this type, see Magnani's thesis [5].

However, it is not clear that this is a proper definition to use in $\mathrm{H}^{1}$. Franchi, Serapioni and Serra Cassano in [2] defined a set $E \subset \mathrm{H}^{n}=\mathrm{C}^{n} \times \mathrm{R}$ to be rectifiable if there are intrinsic $C^{1}$ (in a natural intrinsic sense) functions $u_{i}: \mathrm{H}^{n} \rightarrow \mathrm{R}$ such that

$$
\mathscr{H}^{2 n+1}\left(E \backslash \bigcup_{i=1}^{\infty}\left\{x: u_{i}(x)=0\right\}\right)=0 .
$$

The Hausdorff dimension of $\mathrm{H}^{n}$ is $2 n+2$ so this is a codimension 1 theory. In fact, they developed this theory in more general spaces, see [3], and they used it to establish de Giorgi's theory of sets of finite perimeter in these spaces.

Suppose $E \subset \mathrm{H}^{n}$ is rectifiable in this sense with $\mathscr{H}^{2 n+1}(E)<\infty$. Serra Cassano has shown that then $E$ is rectifiable in the sense of [2] if and only if the restriction measure $\left.\mathscr{H}^{2 n+1}\right|_{E}$ has a unique tangent measure at $\mathscr{H}^{2 n+1}$ almost all points $a \in E$. So this definition seems like a natural one from the point of view we have discussed in this paper.

\section{REFERENCES}

1. Ambrosio, L., and Kirchheim, B., Rectifiable sets in metric and Banach spaces, Math. Ann. 318 (2000), 527-555.

2. Franchi, B., Serapioni, R., and Serra Cassano, F., Rectifiability and perimeter in the Heisenberg group, Math. Ann. 321 (2001), 479-531.

3. Franchi, B., Serapioni, R., and Serra Cassano, F., On the structure of finite perimeter sets in step 2 Carnot groups, J. Geom. Anal. 13 (2003), 421-466.

4. Federer, H., Geometric Measure Theory, Springer-Verlag, 1969. 
5. Magnani, V., Elements of Geometric Measure Theory on Sub-Riemannian Groups, Scuola Normale Superiore, Pisa, Ph.D. thesis, 2002.

6. Mattila, P., Geometry of Sets and Measures in Euclidean Spaces, Cambridge University Press, 1995.

7. Preiss, D., Geometry of Measures in $\mathbf{R}^{n}$ : distribution, rectifiability and densities, Ann. of Math. 125 (1987), 537-643.

DEPARTMENT OF MATHEMATICS AND SATISTICS

FIN-00014 UNIVERSITY OF HELSINKI

FINLAND

E-mail: pertti.mattila@helsinki.fi 